\title{
Microscopic entropy of higher-dimensional nonminimally dressed Lifshitz black holes
}

\author{
Eloy Ayón-Beato, ${ }^{1, *}$ Moisés Bravo-Gaete, ${ }^{2, \dagger}$ Francisco Correa, ${ }^{3, \$}$ \\ Mokhtar Hassaine, ${ }^{4, \S}$ and María Montserrat Juárez-Aubry, ${ }^{5, \|}$ \\ ${ }^{1}$ Departamento de Física, CINVESTAV-IPN, Apartado Postal 14-740, 07000, CDMX, México \\ ${ }^{2}$ Facultad de Ciencias Básicas, Universidad Católica del Maule, Casilla 617, Talca, Chile \\ ${ }^{3}$ Instituto de Ciencias Físicas y Matemáticas, Universidad Austral de Chile, Casilla 567, Valdivia, Chile \\ ${ }^{4}$ Instituto de Matemática y Física, Universidad de Talca, Casilla 747, Talca, Chile \\ ${ }^{5}$ Arkansas State University, Carretera estatal \#100 km. 17.5, Municipio Colón 76270, \\ Querétaro, Querétaro, México
}

(Received 20 May 2019; published 12 August 2019)

\begin{abstract}
In arbitrary dimension, we consider a theory described by the most general quadratic curvature corrections of Einstein gravity together with a self-interacting nonminimally coupled scalar field. This theory is shown to admit five different families of Lifshitz black holes dressed with a nontrivial scalar field. The entropy of these configurations is microscopically computed by means of a higher-dimensional anisotropic Cardy-like formula where the role of the ground state is played by the soliton obtained through a double analytic continuation. This involves calculating the correct expressions for the masses of the higher-dimensional Lifshitz black hole as well as their corresponding soliton. The robustness of this Cardylike formula is checked by showing that the microscopic entropy is in perfect agreement with the gravitational Wald entropy. Consequently, the calculated global charges are compatible with the first law of thermodynamics as well as an anisotropic higher-dimensional version of the Smarr formula. Some of these configurations exist on Lifshitz critical points of the theory where all their extensive thermodynamic quantities vanish.
\end{abstract}

DOI: 10.1103/PhysRevD.100.044024

\section{INTRODUCTION}

Gauge/gravity duality can be extended to nonrelativistic systems by using anisotropic spacetimes. In this context, the archetypal example is the Lifshitz spacetime [1]

$$
d s^{2}=-\frac{r^{2 z}}{l^{2 z}} d t^{2}+\frac{l^{2}}{r^{2}} d r^{2}+\frac{r^{2}}{l^{2}} \sum_{i=1}^{D-2} d x_{i}^{2}
$$

whose main feature is the isometry allowing time and space to scale with different exponents. Here, $z$ is the dynamical critical exponent responsible for the anisotropic scaling characterizing nonrelativistic systems.

\footnotetext{
*ayon-beato-at-fis.cinvestav.mx

mbravo-at-ucm.cl

${ }^{\ddagger}$ francisco.correa-at-uach.cl

${ }^{\S}$ hassaine-at-inst-mat.utalca.cl

"mjuarez-at-astate.edu
}

Published by the American Physical Society under the terms of the Creative Commons Attribution 4.0 International license. Further distribution of this work must maintain attribution to the author(s) and the published article's title, journal citation, and DOI. Funded by SCOAP.
As it was preliminarily emphasized in [1], standard vacuum Einstein gravity cannot allow Lifshitz spacetimes, except in the isotropic case $z=1$ where they turn out to be anti-de Sitter (AdS) spaces. Nevertheless, this problem can be circumvented by considering instead higher-order corrections to gravity theories or by introducing specific matter sources. It then becomes important to find specific gravity models that can accommodate the Lifshitz spacetimes together with their black hole extensions recovering the anisotropic scaling asymptotically. These so-called Lifshitz black holes are supposed to holographically capture the finite-temperature behavior of their strongly correlated nonrelativistic dual systems. New Massive Gravity [2] was one of the first gravity models that was shown to admit an analytic Lifshitz black hole as part of its vacua [3], which is a property that later resulted to be generic for higher-order pure gravity theories in higher dimensions [4]. In presence of specific matter sources, Lifshitz solutions have also been investigated; see, e.g., [5-9]. Also, charged Lifshitz solutions can be engineered through a MaxwellProca model [10], its nonlinear generalization [11] or in the presence of dilaton couplings $[12,13]$.

The relevance of Lifshitz black holes lies in the hope that strongly coupled condensed matter systems can be better 
understood at finite temperature from a holographic point of view. But because of their unconventional asymptotic behavior, these black holes present interesting features which deserve more profound investigations. For example, their thermodynamic properties are usually quite different from those of the isotropic AdS black holes and, in particular, if the solutions are charged; see, e.g., [14,15]. On the other hand, three-dimensional configurations are usually excellent laboratories to investigate important conceptual questions about the gauge/gravity duality. For example, it has been shown that the semiclassical entropy of three-dimensional black holes with Lifshitz asymptotics can be recovered through a Cardy-like formula where the mass of their corresponding Lifshitz solitons explicitly appears, giving a prominent role to these regular configurations [16]. The solitons are obtained from the black holes by means of a double Wick rotation that involves inverting the dynamical critical exponent, as result they enjoy the same sort of uniqueness as the black holes [17]. The robustness of this formula has been successfully tested in a system exhibiting a wide spectrum of Lifshitz configurations as is the case of self-interacting scalar fields nonminimally coupled to New Massive Gravity [18]. Recently, this Cardy-like formula has been extended to higher-dimensional anisotropic black holes [19-21]. In the present work, we pretend to test the validity of this higherdimensional Cardy-like formula by considering again selfinteracting scalar fields, but nonminimally coupled now to the most general quadratic curvature corrections of Einstein gravity in higher dimensions. We hope this study will contribute to highlight the importance of the role played by the soliton in the description of the thermal properties of black holes, and particulary for those with unconventional asymptotic behaviors.

The paper is organized as follows. In the next section we will present the theory, field equations as well as a specific ansatz allowing particular Lifshitz black hole solutions. Using this ansatz in Sec. III, the thermodynamic quantities of interest as entropy, temperature, and mass of the black holes will be preliminarily evaluated together with the mass of the solitons. In Sec. IV, we will explicitly present the first two concrete classes of Lifshitz black holes fitting our ansatz. For these solutions, we will check that their gravitational entropy, calculated with the standard Wald formula, can be correctly reproduced by means of the Cardy-like formula. We analyze separately in Sec. V the other two solutions classes within the ansatz, since they exist at Lifshitz critical points of the theory where all the extensive thermodynamic quantities vanish, trivially satisfying all the thermodynamic relations. In the last section, we show the existence of a fifth class of Lifshitz black hole that is slightly different from the initial working ansatz. For this solution, we also test the robustness of the Cardy-like formula. In all cases we verify the fulfillment of the first law of black hole thermodynamics and the related anisotropic version of the Smarr formula, which point to the correctness of the quasilocal off-shell extension of the AbbottDeser-Tekin formalism that we use to compute global charges. Finally, after some reflections on interesting aspects raised by the new configurations that we include in the conclusions section, we provide several Appendixes for reporting some of the quite involved expressions for the (coupling) constants of the specific solutions.

\section{SETUP OF THE PROBLEM}

In arbitrary dimension $D$, we consider a gravity action given by the most general quadratic curvature corrections of the Einstein-Hilbert action sourced by a self-interacting nonminimally coupled scalar field

$$
S\left[g_{\mu \nu}, \Phi\right]=\int d^{D} x \sqrt{-g}\left(\mathcal{L}_{\mathrm{g}}+\mathcal{L}_{\mathrm{s}}\right),
$$

with

$$
\begin{aligned}
& \mathcal{L}_{\mathrm{g}}=\frac{1}{2 \kappa}\left(R-2 \lambda+\beta_{1} R^{2}+\beta_{2} R_{\alpha \beta} R^{\alpha \beta}+\beta_{3} R_{\alpha \beta \mu \nu} R^{\alpha \beta \mu \nu}\right), \\
& \mathcal{L}_{\mathrm{s}}=-\frac{1}{2} \nabla_{\mu} \Phi \nabla^{\mu} \Phi-\frac{1}{2} \xi R \Phi^{2}-U(\Phi) .
\end{aligned}
$$

The values of the cosmological constant $\lambda$, the coupling constants $\beta_{n}$, the self-interacting potential $U(\Phi)$, and eventually the nonminimal coupling parameter $\xi$ will explicitly depend on the concrete solutions presented in the sections that follow, and in many cases they will be given in the Appendixes. The field equations obtained by varying the action with respect to the metric and the scalar field read

$$
\begin{gathered}
G_{\mu \nu}+\lambda g_{\mu \nu}+K_{\mu \nu}=\kappa T_{\mu \nu}, \\
\square \Phi-\xi R \Phi=\frac{d U(\Phi)}{d \Phi},
\end{gathered}
$$

where we have defined

$$
\begin{aligned}
K_{\mu \nu}= & \left(\beta_{2}+4 \beta_{3}\right) \square R_{\mu \nu}+\frac{1}{2}\left(4 \beta_{1}+\beta_{2}\right) g_{\mu \nu} \square R \\
& -\left(2 \beta_{1}+\beta_{2}+2 \beta_{3}\right) \nabla_{\mu} \nabla_{\nu} R+2 \beta_{3} R_{\mu \gamma \alpha \beta} R_{\nu}{ }^{\gamma \beta \beta} \\
& +2\left(\beta_{2}+2 \beta_{3}\right) R_{\mu \alpha \nu \beta} R^{\alpha \beta}-4 \beta_{3} R_{\mu \alpha} R_{\nu}{ }^{\alpha}+2 \beta_{1} R R_{\mu \nu} \\
& -\frac{1}{2}\left(\beta_{1} R^{2}+\beta_{2} R_{\alpha \beta} R^{\alpha \beta}+\beta_{3} R_{\alpha \beta \gamma \delta} R^{\alpha \beta \gamma \delta}\right) g_{\mu \nu},
\end{aligned}
$$

and the energy-momentum tensor is given by

$$
\begin{aligned}
T_{\mu \nu}= & \nabla_{\mu} \Phi \nabla_{\nu} \Phi-g_{\mu \nu}\left(\frac{1}{2} \nabla_{\sigma} \Phi \nabla^{\sigma} \Phi+U(\Phi)\right) \\
& +\xi\left(g_{\mu \nu} \square-\nabla_{\mu} \nabla_{\nu}+G_{\mu \nu}\right) \Phi^{2} .
\end{aligned}
$$


In order to look for Lifshitz black holes, we will opt for the following ansatz

$$
\begin{aligned}
d s^{2} & =-\frac{r^{2 z}}{l^{2 z}} f(r) d t^{2}+\frac{l^{2}}{r^{2}} \frac{d r^{2}}{f(r)}+\frac{r^{2}}{l^{2}} \sum_{i=1}^{D-2} d x_{i}^{2}, \\
\Phi & =\Phi(r),
\end{aligned}
$$

where the structural metric function satisfies the boundary condition $\lim _{r \rightarrow \infty} f(r)=1$, ensuring the metric to reproduce the Lifshitz asymptotics (1). As shown below, four of the five classes of Lifshitz solutions that will be presented can be generically parametrized as

$$
f(r)=1-\left(\frac{r_{h}}{r}\right)^{\chi}, \quad \Phi(r)=\Phi_{0}\left(\frac{r_{h}}{r}\right)^{\frac{\chi}{2}},
$$

where $\chi$ is a non-negative decay exponent modulating the Lifshitz asymptotics, $r_{h}$ denotes the location of the horizon, and $\Phi_{0}$ characterizes the positive strength of the field. The positivity of the scalar field can be explained from the fact that for these four classes of solutions, the discrete transformation $\Phi \mapsto-\Phi$ will be a symmetry of the problem. The ansatz (4)-(5) is also motivated by the fact that for $\Phi_{0}=0$ most of the vacuum Lifshitz black hole solutions known for the theory have precisely this form [4], which also occurs for their charged extensions [14]. The remaining solutions belong to a different class where the structural metric function involves two different radial powers and will be presented in Sec. VI.

One of the main aims of this work is to confirm the importance of the role played by the gravitational soliton for the thermal properties of the Lifshitz black holes. In order to achieve this task correctly, we will need the Lifshitz soliton counterparts of the black holes (4)-(5). The solitons will be generically described by the following metric

$d \bar{s}^{2}=-\frac{\bar{r}^{2}}{l^{2}} d \bar{t}^{2}+\frac{l^{2}}{\bar{r}^{2}} \frac{d \bar{r}^{2}}{f(\bar{r})}+\frac{\bar{r}^{2 z}}{l^{2 z}} f(\bar{r}) d \bar{x}_{1}^{2}+\frac{\bar{r}^{2}}{l^{2}} \sum_{i=2}^{D-2} d \bar{x}_{i}^{2}$,

with

$f(\bar{r})=1-\left[\left(\frac{2}{\chi}\right)^{1 / z} \frac{l}{\bar{r}}\right]^{\chi}, \quad \Phi(\bar{r})=\Phi_{0}\left[\left(\frac{2}{\chi}\right)^{1 / z} \frac{l}{\bar{r}}\right]^{\frac{\chi}{2}}$.

The solitons are obtained from the black holes by means of a double Wick rotation $\bar{t}=-i x_{1}$ and $\bar{x}_{1}=-i t$ supplemented by an adjustment of the horizon location

$$
r_{h}=l\left(\frac{2}{\chi}\right)^{\frac{1}{2}}
$$

which ensures the correct identification of its Euclidean version.
The configurations that will be described below are fully determined in terms of three parameters, namely, the decay exponent $\chi$, the strength $\Phi_{0}$, and the dynamical exponent $z$. In order to simplify the discussion, we start by evaluating first the formulas of interest as the entropy, temperature and mass of the black hole configurations as well as the mass of the solitons for generic values of these constants, i.e., not for those that actually satisfy all the system constraints. The precise thermodynamic quantities are given later for each genuine solution with the help of these formulas.

\section{PRELIMINARY THERMODYNAMIC QUANTITIES}

First of all, the Wald entropy formula [22] for action (2) evaluated in the black hole ansatz (4)-(5) generically reads

$$
\mathcal{S}_{\mathrm{W}}=\frac{2 \pi \Upsilon}{\kappa}\left(\frac{r_{h}}{l}\right)^{D-2} \Omega_{D-2},
$$

where $\Omega_{D-2}$ represents the finite volume of the $(D-2)$ dimensional planar base manifold and the coefficient

$$
\begin{aligned}
\Upsilon \equiv & -\left.\kappa P^{a b c d} \varepsilon_{a b} \varepsilon_{c d}\right|_{r=r_{h}} \\
= & 1-\kappa \xi \Phi_{0}^{2}+\frac{\chi}{l^{2}}\left[2(\chi-3 z-2 D+4) \beta_{1}\right. \\
& \left.+(\chi-3 z-D+2) \beta_{2}+2(\chi-3 z) \beta_{3}\right],
\end{aligned}
$$

with $P^{a b c d} \equiv \partial\left(\mathcal{L}_{\mathrm{g}}+\mathcal{L}_{\mathrm{s}}\right) / \partial R_{a b c d}$ measures how the theory departs from the behavior of standard gravity, whose areal interpretation of black holes entropy forces $\Upsilon=1$. On the other hand, their temperature is given by

$$
T=\frac{1}{4 \pi} \frac{r_{h}^{z+1}}{l^{z+1}} f^{\prime}\left(r_{h}\right)=\frac{\chi}{4 \pi l}\left(\frac{r_{h}}{l}\right)^{z} .
$$

In order to compute the masses of the black hole and soliton configurations defined in Eqs. (4)-(7), we will opt for the quasilocal formalism as defined in $[23,24]$. Notice that this formalism has proved to be well suited for correctly computing the masses of black holes of higherorder gravity theories with rather unconventional asymptotics; see, e.g., [18]. The quasilocal formalism is based on an off-shell prescription [23] for the Abbott-Deser-Tekin potential [25] which allows the following concise expression for the conserved charge associated to a Killing vector field $k$

$Q(k)=\int_{\mathcal{B}} d^{D-2} x_{\mu \nu}\left(\Delta N^{\mu \nu}(k)-2 k^{[\mu} \int_{0}^{1} d s \Theta^{\nu]}(k \mid s)\right)$,

where $s$ is a parameter interpolating between the solution of interest at $s=1$ and the asymptotic one at $s=0$, the difference between their off-shell Noether potentials is 
denoted by $\Delta N^{\mu \nu}(k) \equiv N_{s=1}^{\mu \nu}(k)-N_{s=0}^{\mu \nu}(k)$ and $\Theta^{\nu}$ is the surface term arising after varying the action. In the present case, these tensors are given by

$$
\begin{aligned}
\Theta^{\mu}= & 2 \sqrt{-g}\left(P^{\mu(\alpha \beta) \gamma} \nabla_{\gamma} \delta g_{\alpha \beta}-\delta g_{\alpha \beta} \nabla_{\gamma} P^{\mu(\alpha \beta) \gamma}\right. \\
& \left.+\frac{1}{2} \frac{\partial \mathcal{L}_{\mathrm{s}}}{\partial\left(\partial_{\mu} \Phi\right)} \delta \Phi\right), \\
N^{\mu \nu}= & 2 \sqrt{-g}\left(P^{\mu \nu \rho \sigma} \nabla_{\rho} k_{\sigma}-2 k_{\sigma} \nabla_{\rho} P^{\mu \nu \rho \sigma}\right) .
\end{aligned}
$$

For a timelike Killing vector field, $\partial_{t}=k^{\mu} \partial_{\mu}$, the evaluation of the mass formula for action (2) in the black hole ansatz (4)-(5) gives rise to the expression

$$
\begin{aligned}
\mathcal{M}_{\mathrm{bh}}(k)= & \left\{-4 \Psi_{1}+\kappa \Phi_{0}^{2}[2(2 \chi+2 z-D+2) \xi-\chi]\right\} \\
& \times\left(\frac{r_{h}}{l}\right)^{2 \chi}\left(\frac{l}{r}\right)^{2 \chi-z-D+2} \frac{\Omega_{D-2}}{8 \kappa l} \\
& +\left\{2 \Psi_{2}-\kappa \Phi_{0}^{2}[4(\chi+z) \xi-\chi]\right\} \\
& \times\left(\frac{r_{h}}{l}\right)^{\chi}\left(\frac{l}{r}\right)^{\chi-z-D+2} \frac{\Omega_{D-2}}{4 \kappa l}
\end{aligned}
$$

where $\Psi_{1}$ and $\Psi_{2}$ are two dimensionless linear combinations of the squared corrections coupling constants reported in Appendix A. For the soliton ansatz (4)-(7) with timelike Killing vector field $\partial_{\bar{t}}=k^{\mu} \partial_{\mu}$ the mass formula reads

$$
\begin{aligned}
\mathcal{M}_{\mathrm{sol}}(k)= & \left\{-4 \Xi_{1}+\kappa \Phi_{0}^{2}[2(4 \chi-2 z-D+6) \xi-\chi]\right\} \\
& \times\left(\frac{2}{\chi}\right)^{2 \chi / z}\left(\frac{l}{\bar{r}}\right)^{2 \chi-z-D+2} \frac{\Omega_{D-2}}{8 \kappa l} \\
& +\left\{2 \Xi_{2}-\kappa \Phi_{0}^{2}[4(\chi+1) \xi-\chi]\right\} \\
& \times\left(\frac{2}{\chi}\right)^{\chi / z}\left(\frac{l}{\bar{r}}\right)^{\chi-z-D+2} \frac{\Omega_{D-2}}{4 \kappa l}
\end{aligned}
$$

where the dimensionless coupling constants combinations $\Xi_{1}$ and $\Xi_{2}$ are also defined in Appendix A. For actual solutions, the mass expressions (11) and (12) must be global charges and cannot depend on the radial coordinates $r$ and $\bar{r}$, respectively. Interestingly, this imposes constraints on the constants $z, \chi$, and $\Phi_{0}$ giving indications on the possible solutions within the ansatz; concretely, only two families of decay exponents $\chi$ are possible since they are the only ones giving rise to nontrivial global charge masses.

In what follows, we will report four different classes of Lifshitz black hole solutions fitting our ansatz (4)-(5). For each solution, we will check that its gravitational Wald entropy (8) is correctly reproduced by means of a higherdimensional anisotropic Cardy-like formula [21] given by

$$
\mathcal{S}_{\mathrm{C}}=\frac{2 \pi l(z+D-2)}{D-2}\left(-\frac{(D-2) \mathcal{M}_{\mathrm{sol}}}{z}\right)^{\frac{z}{z+D-2}} \mathcal{M}_{\mathrm{bh}^{\frac{D-2}{z+D-2}}}
$$

This expression is the higher-dimensional extension of the one obtained for two-dimensional Lifshitz field theory [16]. Here we have used the notation $\mathcal{S}_{\mathrm{C}}$ for the microscopic entropy in order to reflect that the anisotropic Cardy-like expression is a priori different from the gravitational Wald entropy (8). Nevertheless, as shown below, both entropies will coincide for the different classes of solutions reported. For completeness, we will also verify that the first law of black hole thermodynamics,

$$
d \mathcal{M}_{\mathrm{bh}}=T d \mathcal{S}_{\mathrm{W}}
$$

consistently holds for each Lifshitz black hole solution.

\section{NONCRITICAL LIFSHITZ BLACK HOLES}

Here, we will present the first two classes of solutions that fit within ansatz (4)-(5) and compute their definitive thermodynamic quantities through the preliminary formulas derived in the previous section. All of them will turn out to be nontrivial, unlike the critical cases that we leave for the next section, which explains why we call the following solutions noncritical. For each solution, we will corroborate that their Wald entropy can be reproduced from the anisotropic Cardy-like formula (13). We also show that the fulfillment of the first law (14) for all is linked with a higher-dimensional anisotropic version of the Smarr formula.

\section{A. Class with arbitrary dynamical exponent and arbitrary nonminimal coupling parameter}

The first family of solutions is obtained for the standard potential where a mass term is supplemented by a quartic interaction and exists for arbitrary values of the dynamical exponent $z$ and of the nonminimal coupling parameter $\xi$. Because of cumbersome formulas, the concrete form of the potential and the parametrizations obeying the different coupling constants as well as the cosmological constant are reported in Appendix B. Its line element and the nontrivial scalar field are given by

$$
\begin{aligned}
d s^{2}= & -\left(\frac{r}{l}\right)^{2 z}\left[1-\left(\frac{r_{h}}{r}\right)^{(z+D-2) / 2}\right] d t^{2} \\
& +\frac{l^{2}}{r^{2}}\left[1-\left(\frac{r_{h}}{r}\right)^{(z+D-2) / 2}\right]^{-1} d r^{2}+\frac{r^{2}}{l^{2}} \sum_{i=1}^{D-2} d x_{i}^{2} \\
\Phi(r)= & \left(\frac { 1 } { \kappa l ^ { 2 } P _ { 5 } ( z ; \xi ) } \left\{\left[3 z^{2}+(D+2)(D-2)\right] P_{3}(z) l^{2}\right.\right. \\
& \left.\left.-2(D-3)(D-4)(z+D-2) P_{4}(z) \beta_{3}\right\}\right)^{1 / 2} \\
& \times\left(\frac{r_{h}}{r}\right)^{(z+D-2) / 4},
\end{aligned}
$$


where the polynomials $P_{n}$ are also defined in Appendix B together with the remaining details of the solution.

This solution is obtained from the proposed ansatz (5) by using one of the only two decay exponents allowing a welldefined Lifshitz mass, namely, $\chi=(z+D-2) / 2$. The result is the higher-dimensional lifting from $D=3$ of the black hole family with Lifshitz decay $(z+1) / 2$ originally derived in Ref. [8] for New Massive Gravity, whose thermodynamics was studied in Ref. [18]. It is interesting to notice that the above higher-dimensional line element has been previously obtained also as a vacuum solution in [4], but for a more restrictive election of the coupling constants. The vacuum limit of [4] is easily recovered by fixing the coupling constant $\beta_{3}$ in solution (15) in order to obtain a vanishing scalar strength. In this sense the present solution is also a generalization of the one of [4] allowing the same black hole to be dressed by a self-interacting nonminimally coupled scalar field. Notice that the existence of nonminimal coupling is not imperative since the solution still exists in the limit $\xi=0$.

The thermodynamic properties of the lifted configuration follow from the following expressions, first, the preliminary formula (8) gives the Wald entropy

$$
\mathcal{S}_{\mathrm{W}}=\frac{2 \pi \Upsilon_{1}}{\kappa}\left(\frac{r_{h}}{l}\right)^{D-2} \Omega_{D-2}
$$

where the dimensionless coefficient $\Upsilon_{1}$, depending on the free coupling constants, is mutual to all the extensive thermodynamic quantities associated with the solution and is defined in Appendix D. The Hawking temperature (9) in this case reads

$$
T=\frac{z+D-2}{8 \pi l}\left(\frac{r_{h}}{l}\right)^{z}
$$

The formulas (11) and (12) lead to the Lifshitz black hole and soliton masses, respectively,

$$
\begin{aligned}
\mathcal{M}_{\mathrm{bh}} & =\frac{(D-2) \Upsilon_{1}}{4 \kappa}\left(\frac{r_{h}}{l}\right)^{z+D-2} \frac{\Omega_{D-2}}{l}, \\
\mathcal{M}_{\mathrm{sol}} & =-\frac{z \Upsilon_{1}}{4 \kappa}\left(\frac{4}{z+D-2}\right)^{\frac{z+D-2}{z}} \frac{\Omega_{D-2}}{l} .
\end{aligned}
$$

Now, it is straightforward to verify that the Cardy-like formula (13) for the entropy correctly reproduces the gravitational Wald entropy, that is $\mathcal{S}_{\mathrm{W}}=\mathcal{S}_{\mathrm{C}}$.

Another interesting feature of these thermodynamic quantities is that they obey the following anisotropic higher-dimensional version of the Smarr formula [26]

$$
\mathcal{M}_{\mathrm{bh}}=\frac{D-2}{z+D-2} T \mathcal{S}_{\mathrm{W}}
$$

which in fact is not unexpected since it is indispensable for they consequently respect the first law (14).

For the other decay exponent compatible with a welldefined mass, $\chi=z+D-2$, it happens that one obtains a vanishing mass, as will be exhibited in Sec. V. However, there is an exception for the critical exponent $z=D$; this is the solution we present below.

\section{B. Solution with dynamical exponent $z=D$}

The second family of Lifshitz black hole solutions exists for a dynamical exponent $z=D$ and for a nonminimal coupling parameter $\xi<(D-1) /(5 D-2)$,

$$
\begin{aligned}
d s^{2}= & -\frac{r^{2 D}}{l^{2 D}}\left[1-\left(\frac{r_{h}}{r}\right)^{2(D-1)}\right] d t^{2} \\
& +\frac{l^{2}}{r^{2}}\left[1-\left(\frac{r_{h}}{r}\right)^{2(D-1)}\right]^{-1} d r^{2}+\frac{r^{2}}{l^{2}} \sum_{i=1}^{D-2} d x_{i}^{2}, \\
\Phi(r)= & \frac{1}{\sqrt{\kappa}} \sqrt{\frac{(D-2)}{D-1-(5 D-2) \xi}\left(\frac{r_{h}}{r}\right)^{D-1}} .
\end{aligned}
$$

In this case, the self-interacting potential is also given by a mass term plus a $\Phi^{4}$ interaction and the coupling constants $\beta_{1}$ and $\beta_{3}$ are arbitrary

$$
\begin{aligned}
U(\Phi)= & -\frac{(D-1)[D-1-(5 D-2) \xi]}{4(D-2) l^{2}} \\
& \times\left\{2(D-2) \Phi^{2}+[D-1-(D-2) \xi] \kappa \Phi^{4}\right\}, \\
\lambda= & \frac{(D-1)\left[8 D(D-3)(D-4) \beta_{3}-(5 D-2) l^{2}\right]}{4 l^{4}}, \\
\beta_{2}= & -\frac{2(5 D-2)(D-1) \beta_{1}+4(2 D+1) \beta_{3}-l^{2}}{2(D+2)(D-1)} .
\end{aligned}
$$

We remark that the restriction on the nonminimal coupling parameter consistently includes the minimal case $\xi=0$. This solution consistently fits the working ansatz (5) within the other admissible family of decay exponents $\chi=$ $z+D-2=2(D-1)$ when the critical exponent takes the value $z=D$. Its Hawking temperature becomes

$$
T=\frac{(D-1)}{2 \pi l}\left(\frac{r_{h}}{l}\right)^{D}
$$

while the entropy together with the masses of the black hole and its soliton counterpart are given by 


$$
\begin{aligned}
\mathcal{S}_{\mathrm{W}} & =\frac{2 \pi \Upsilon_{2}}{\kappa}\left(\frac{r_{h}}{l}\right)^{D-2} \Omega_{D-2}, \\
\mathcal{M}_{\mathrm{bh}} & =\frac{(D-2) \Upsilon_{2}}{2 \kappa}\left(\frac{r_{h}}{l}\right)^{2(D-1)} \frac{\Omega_{D-2}}{l}, \\
\mathcal{M}_{\mathrm{sol}} & =-\frac{D \Upsilon_{2}}{2 \kappa}\left(\frac{1}{D-1}\right)^{\frac{2(D-1)}{D}} \frac{\Omega_{D-2}}{l},
\end{aligned}
$$

where the mutual extensive coefficient is again defined in Appendix D. As before, one can check the validity of the first law and the Cardy-like formula (13), as well as of the Smarr formula (20).

\section{CRITICAL LIFSHITZ BLACK HOLES}

In the previous section it was shown that the extensive thermodynamic quantities share the same coefficient $\Upsilon$, whose general definition is given in (8b). Consequently, the points in the parameter space of the theory where $\Upsilon=0$ will give rise to Lifshitz black holes with vanishing extensive thermodynamic quantities. Notice that in the absence of a scalar field and for isotropic scaling $z=1$ (i.e., for AdS black holes, whose standard decay is $\chi=z+D-2=D-1$ to be compatible with the AdS mass) this is precisely the case in $D=4$ of the so-called critical gravity point [27,28], where $\beta_{2}=-3 \beta_{1}=-l^{2} / 2$ giving

$$
\Upsilon=1-\frac{6}{l^{2}}\left(4 \beta_{1}+\beta_{2}\right)=0 .
$$

As has been highlighted, e.g., in [29], the common criteria that allows to generically define critical gravity points for any theory is that the entropy as well as the global charges of their black holes vanish on this specific region of the parameter space. In this sense, $\Upsilon=0$ defines Lifshitz critical points of the examined theory. In this section we present two other families fitting our ansatz (5) which are examples of critical Lifshitz black holes, since they exist at points where $\Upsilon=0$, sharing the peculiarity of having vanishing Wald entropy and masses. They trivially satisfy the first law of thermodynamics as well as the Cardy-like formula since $\mathcal{S}_{\mathrm{W}}=0=\mathcal{S}_{C}$.

The first critical solution is obtained by choosing the other decay exponent compatible with a well-defined Lifshitz mass $\chi=z+D-2$, which gives

$$
\begin{aligned}
d s^{2}= & -\frac{r^{2 z}}{l^{2 z}}\left[1-\left(\frac{r_{h}}{r}\right)^{z+D-2}\right] d t^{2} \\
& +\frac{l^{2}}{r^{2}}\left[1-\left(\frac{r_{h}}{r}\right)^{z+D-2}\right]^{-1} d r^{2}+\frac{r^{2}}{l^{2}} \sum_{i=1}^{D-2} d x_{i}^{2}, \\
\Phi(r)= & 2 \sqrt{\frac{(D-2)(z-1)}{\kappa P_{2}(z ; \xi)}\left(\frac{r_{h}}{r}\right)^{\frac{z+D-2}{2}},}
\end{aligned}
$$

where the second grade polynomial in the critical exponent at the denominator of the scalar strength is defined as

$$
P_{2}(z ; \xi) \equiv(z+D-2)^{2}-4\left[2 z^{2}+(D-2)(2 z+D-1)\right] \xi .
$$

This solution is the higher-dimensional lifting of the family with Lifshitz decay $z+1$ obtained in Ref. [8]. The selfinteraction potential supporting the solution and the values of the coupling constants are extended as

$$
\begin{aligned}
U(\Phi)= & -\frac{P_{2}(z ; \xi)}{16 l^{2}}\left[2 \Phi^{2}+\left(\frac{(z+D-2)^{2}}{4(D-2)(z-1)}-\xi\right) \kappa \Phi^{4}\right], \\
\beta_{1}= & \frac{(D-2) z^{2}+2(D-3) z-(D-2)^{2}}{(D-2)(z-1)(z+D-2)} \beta_{3} \\
& +\frac{(1-4 \xi) l^{2}}{2 P_{2}(z ; \xi)}, \\
-\beta_{2}= & \frac{(D-2)\left(4 z^{2}-D^{2}+3 D-4\right)+2 D(D-3) z}{(D-2)(z-1)(z+D-2)} \beta_{3} \\
& +\frac{l^{2}}{2 P_{2}(z ; \xi)},
\end{aligned}
$$

while the cosmological constant $\lambda$ takes the same expression given in Appendix B. It is straightforward to check by means of formulas (11)-(12) that the masses of the black hole and its soliton counterpart are zero, which is a property also shared by the Wald entropy (8).

The other critical solution is obtained for the decay exponent $\chi=2(z-1)$ which does not give a global charge in general. However, together with its accompanying coupling constants they exactly cancel the coefficients in front of the decaying powers that prevent the mass formula from becoming a conserved charge, causing its vanishing at the same time. The resulting solution is

$$
\begin{aligned}
d s^{2}= & -\left(\frac{r}{l}\right)^{2 z}\left[1-\left(\frac{r_{h}}{r}\right)^{2(z-1)}\right] d t^{2} \\
& +\frac{l^{2}}{r^{2}}\left[1-\left(\frac{r_{h}}{r}\right)^{2(z-1)}\right]^{-1} d r^{2}+\frac{r^{2}}{l^{2}} \sum_{i=1}^{D-2} d x_{i}^{2}, \\
\Phi(r)= & \left(\frac { 1 } { \kappa l ^ { 2 } \tilde { P } _ { 2 } ( z ; \xi ) } \left[4(D-3)(D-4) z(z-D) \beta_{3}\right.\right. \\
& \left.\left.-(D-1)(2 z-D-2) l^{2}\right]\right)^{1 / 2}\left(\frac{r_{h}}{r}\right)^{z-1},
\end{aligned}
$$

where the critical exponent polynomial is given by

$$
\tilde{P}_{2}(z ; \xi) \equiv\left[2 z^{2}+(D-2)(2 z+D-1)\right] \xi-(D-1)(z-1),
$$

and the specific parametrizations of the coupling constants together with the cosmological one are presented in Appendix C. 
It is interesting to emphasize that this line element corresponds to the other of the vacuum solutions previously obtained in [4] for the same theory, but with a more restrictive choice of the coupling constants. Notice that if we fix the coupling constant $\beta_{3}$ by demanding the vanishing of the scalar strength we recover the black hole without a scalar field of [4]. In other words, this solution generalizes the other vacuum example of a higher-dimensional Lifshitz black hole by dressing it with a self-interacting nonminimally coupled scalar field.

All the previous solutions exist for generic values of the nonminimal coupling parameter that include the minimal case $\xi=0$ in particular. In the next section we provide an example which is necessarily nonminimal, but this entails going beyond ansatz (5).

\section{LAST CLASS OF LIFSHITZ BLACK HOLES}

The fifth class of Lifshitz black holes that does not fit within our ansatz (5) has a fixed value of the dynamical exponent $z=D$ and is valid also for a precise value of the nonminimal coupling parameter

$$
\xi=\frac{(2 D-1)(D-1)}{2\left(3 D^{2}-2 D+4\right)(D+1)} .
$$

The configuration in question reads

$$
\begin{aligned}
d s^{2}= & -\frac{r^{2 D}}{l^{2 D}}\left[1-M\left(\frac{l}{r}\right)^{2(D-1)}-\alpha \sqrt{M}\left(\frac{l}{r}\right)^{D-1}\right] d t^{2} \\
& +\frac{l^{2}}{r^{2}}\left[1-M\left(\frac{l}{r}\right)^{2(D-1)}-\alpha \sqrt{M}\left(\frac{l}{r}\right)^{D-1}\right]^{-1} d r^{2} \\
& +\frac{r^{2}}{l^{2}} \sum_{i=1}^{D-2} d x_{i}^{2}, \\
\Phi(r)= & \sqrt{\frac{2(D+1)\left(3 D^{2}-2 D+4\right) M}{\kappa(D-1) P_{2}(D)}\left(\frac{l}{r}\right)^{D-1}},
\end{aligned}
$$

where $\alpha$ is a coupling constant appearing in the potential and $P_{2}(D)=6 D^{2}-16 D-1$. Indeed, this solution exists provided that the potential and the coupling constants are given by

$$
\begin{aligned}
U(\Phi)= & -\frac{(D-2)(D-1)^{2} P_{2}(D)}{4(D+1)\left(3 D^{2}-2 D+4\right) l^{2}} \Phi^{2} \\
& -\frac{\alpha(2 D-1)}{2 l^{2}} \sqrt{\frac{(D-1)^{5} P_{2}(D) \kappa}{2(D+1)\left(3 D^{2}-2 D+4\right)^{3}}} \Phi^{3} \\
& -\frac{(D-1)^{3} P_{2}(D)\left(D^{3}-4 D^{2}+19 D+2\right) \kappa}{16(D+1)^{2}\left(3 D^{2}-2 D+4\right)^{2} l^{2}} \Phi^{4}, \\
\beta_{1}= & \frac{\left(2 D^{3}-6 D^{2}+25 D+3\right) l^{2}}{2(D-4)(D-1)^{2} P_{2}(D)},
\end{aligned}
$$

$$
\begin{aligned}
& \beta_{2}=-\frac{2(D+1)\left(D^{2}+3 D-1\right) l^{2}}{(D-4)(D-1)^{2} P_{2}(D)}, \\
& \beta_{3}=\frac{3(D+1) l^{2}}{2(D-4) P_{2}(D)}, \\
& \lambda=-\frac{(D-1)(D-2)\left(18 D^{2}-32 D-1\right)}{4 P_{2}(D) l^{2}} .
\end{aligned}
$$

The event horizon of this black hole is located at the radius

$$
r_{h}^{D-1}=l^{D-1} \frac{\sqrt{M}}{2}\left(\alpha+\sqrt{\alpha^{2}+4}\right),
$$

and in terms of this radius the quantities of interest to corroborate the first law (14), together with the validity of the Cardy-like (13) and Smarr (20) formulas, are given by

$$
\begin{aligned}
\mathcal{S}_{\mathrm{W}} & =\frac{2 \pi \Upsilon_{3}}{\kappa}\left(\frac{r_{h}}{l}\right)^{D-2} \Omega_{D-2}, \\
T & =\frac{(D-1) \sqrt{\alpha^{2}+4}}{2 \pi l\left(\alpha+\sqrt{\alpha^{2}+4}\right)}\left(\frac{r_{h}}{l}\right)^{D}, \\
\mathcal{M}_{\mathrm{bh}} & =\frac{(D-2) \sqrt{\alpha^{2}+4} \Upsilon_{3}}{2 \kappa\left(\alpha+\sqrt{\alpha^{2}+4}\right)}\left(\frac{r_{h}}{l}\right)^{2(D-1)} \frac{\Omega_{D-2}}{l}, \\
\mathcal{M}_{\mathrm{sol}} & =\frac{-D \sqrt{\alpha^{2}+4} \Upsilon_{3}}{2 \kappa\left(\alpha+\sqrt{\alpha^{2}+4}\right)}\left(\frac{\alpha+\sqrt{\alpha^{2}+4}}{(D-1) \sqrt{\alpha^{2}+4}}\right)^{\frac{2(D-1)}{D}} \frac{\Omega_{D-2}}{l},
\end{aligned}
$$

where the extensive coefficient is expressed as all the previous ones in Appendix D.

\section{CONCLUSION}

Here, we have extended the work on dressed Lifshitz black holes done in three dimensions in the case of a scalar field nonminimally coupled to New Massive Gravity [18]. Indeed, we have considered a gravity theory given by the most general quadratic curvature corrections to Einstein gravity supplemented by a source action describing a selfinteracting nonminimally coupled scalar field. For this theory, we have presented five different classes of Lifshitz black hole solutions. Each solution is specified with a particular self-interacting potential and certain parametrization of the cosmological constant and the coupling constants $\beta_{n}$ accompanying the different quadratic invariants.

Interestingly, some of the obtained solutions describe Lifshitz black hole backgrounds that were known previously as part of the vacuum of the studied theories [4], but for more restrictive elections of the coupling constants. These restrictions are recovered from the presented solutions in the limit of a vanishing scalar strength. Hence, the new configurations constitute generalizations of these vacuum higher-dimensional Lifshitz black holes that now turn out to be dressed by self-interacting nonminimally coupled scalar fields. 
We would like to stress that our work constitutes a new example that underlines the importance played by the gravitational solitons in order to describe the thermal properties of black holes with (un)usual asymptotics, since they are indispensable to write the Cardy-like microscopic entropy formula. In this spirit, it will be desirable to keep exploring this issue from the holographic point of view. In particular, a promising work to be done will consist of identifying or interpreting the role of the soliton in the field theory side.

Another interesting aspect that has to do with these solutions concerns the Smarr formula [26]. Indeed, since all the solutions reported here verify the higher-dimensional anisotropic Cardy-like formula (13) as well as the first law of thermodynamics (14), they will also satisfy an anisotropic higher-dimensional version of the Smarr formula (20). This last formula is in perfect accordance with the one discussed, e.g., in Ref. [30] for different theories admitting Lifshitz black holes. In fact, the Smarr formula is just a reflection of the proportionality between the entropy and the black hole mass, a proportionality observed by all the extensive thermodynamics quantities through the same coefficient $\Upsilon$, generically defined in (8b). For example, putting together the Smarr formula (20) and the Cardy-like one (13) it follows the proportionality between the masses of the black holes and their corresponding solitons

$$
\mathcal{M}_{\mathrm{bh}}=-\frac{D-2}{z}(2 \pi l T)^{\frac{z+D-2}{z}} \mathcal{M}_{\mathrm{sol}},
$$

which is checked in all the new solutions.

Consequently, the condition that allows identifying Lifshitz critical points of the theory where all the extensive thermodynamics quantities related to Lifshitz black holes vanish is $\Upsilon=0$. For example, in the absence of a scalar field the related condition is satisfied in the isotropic AdS case $(z=1)$ for the four-dimensional critical gravity point $[27,28]$. Two of the new obtained configurations exist just within the identified anisotropic points, providing examples of Lifshitz critical black holes. It will be interesting to study if their perturbations also have zero energy, with this additional criterion there will be no doubt that these points define well-behaved theories as in the isotropic case.

It is evident that the emergence of the solutions presented here is essentially due to the higher-order nature of the gravity theory together with the nonminimal coupling of the scalar field to this gravity through the conventional term $R \Phi^{2}$. One eventually can pursue the exploration on this issue by studying other Lifshitz black hole solutions that may arise from other nonminimal couplings such as those recently put in the spotlight through the Horndeski Lagrangian [31].

\section{ACKNOWLEDGMENTS}

We thank J. Oliva for his contribution at the initial stage of this work and R. Olea for valuable comments. This work has been partially funded by Grants No. A1-S-11548 from
Conacyt and No. 1171475 from Fondecyt. M. B. is supported by Grant Conicyt/Programa Fondecyt de Iniciación en Investigación No. 11170037. F. C. is supported by the Alexander von Humboldt Foundation and is grateful for the warm hospitality at Leibniz Universität Hannover.

\section{APPENDIX A: EXPRESSIONS FOR THE COEFFICIENTS OF THE MASS FORMULAS (11) AND (12)}

The dimensionless combinations of coupling constants appearing in the black hole mass formula (11) are

$$
\begin{aligned}
l^{2} \Psi_{1}= & (2 \chi+2 z-D+2)\left[\chi^{2}-(3 z+2 D-4) \chi+2 z^{2}\right. \\
& +(D-2)(2 z+D-1)] \beta_{1}+\left\{\chi^{3}-2(z+D-2) \chi^{2}\right. \\
& -\left[z^{2}-(D-2)(3 z+D-2)\right] \chi+(2 z-D+2) \\
& \left.\times\left(z^{2}+D-2\right)\right\} \beta_{2}+\left\{2 \chi^{3}-(4 z+3 D-6) \chi^{2}\right. \\
& -\left[2 z^{2}-(D-2)(9 z-2)\right] \chi+4 z^{3} \\
& \left.-2(D-2)\left(3 z^{2}-3 z+1\right)\right\} \beta_{3}, \\
l^{2} \Psi_{2}= & 2\left\{2 \chi^{3}-4(z+D-2) \chi^{2}-2\left[z^{2}-(D-1)(D-2)\right] \chi\right. \\
& \left.+(2 z-D+2)\left[2 z^{2}+(D-2)(2 z+D-1)\right]\right\} \beta_{1} \\
& +\left\{2 \chi^{3}-(4 z+3 D-6) \chi^{2}-\left[2 z^{2}-(D-2)\right.\right. \\
& \left.\times(3 z+D-2)] \chi+2(2 z-D+2)\left(z^{2}+D-2\right)\right\} \beta_{2} \\
& +4\left\{\chi^{3}-(2 z+D-2) \chi^{2}-\left[z^{2}-(D-2)(3 z-1)\right] \chi\right. \\
& \left.+2 z^{3}-(D-2)\left(3 z^{2}-3 z+1\right)\right\} \beta_{3}+(D-2) l^{2} .
\end{aligned}
$$

The corresponding dimensionless combinations appearing in turn in the soliton mass formula (12) will be

$$
\begin{aligned}
l^{2} \Xi_{1}= & (4 \chi-2 z-D+6)\left[\chi^{2}-(3 z+2 D-4) \chi+2 z^{2}\right. \\
& +(D-2)(2 z+D-1)] \beta_{1}+\left\{\chi^{3}-2(2 z+D-2) \chi^{2}\right. \\
& +\left[5 z^{2}+(3 D-10) z+D(D-2)\right] \chi \\
& \left.-(2 z+D-6)\left(z^{2}+D-2\right)\right\} \beta_{2}-\left\{(2 z-D+6) \chi^{2}\right. \\
& -\left[6 z^{2}-(3 D-2) z+6 D-8\right] \chi \\
& \left.+4 z^{3}-2(D+2) z(z-1)+2 D-12\right\} \beta_{3}, \\
l^{2} \Xi_{2}= & 2\left\{2 \chi^{3}-2(3 z+2 D-5) \chi^{2}+\left[6 z^{2}+6(D-3) z\right.\right. \\
& +(D-2)(3 D-7)] \chi-(2 z+D-6)\left[2 z^{2}\right. \\
& +(D-2)(2 z+D-1)]\} \beta_{1}+\left\{\chi^{3}-(3 z+2 D-4) \chi^{2}\right. \\
& +\left[4 z^{2}+(3 D-10) z+D(D-2)\right] \chi-2(2 z+D-6) \\
& \left.\times\left(z^{2}+D-2\right)\right\} \beta_{2}-4\left[\chi^{2}-\left(z^{2}-z+D-1\right) \chi+2 z^{3}\right. \\
& -(D+2) z(z-1)+D-6] \beta_{3}-(\chi-2 z-D+4) l^{2} .
\end{aligned}
$$




\section{APPENDIX B: PARAMETERS ASSOCIATED TO THE FIRST CLASS OF SOLUTIONS (15)}

The potential associated to the first class of the solutions of subsection IVA reads

$$
\begin{aligned}
U(\Phi)= & \frac{16\left[2 z^{2}+(D-2)(2 z+D-1)\right] \xi-3(z+D-2)^{2}}{32 l^{2}} \Phi^{2}+\left\{4\left[3 z^{2}+(D+2)(D-2)\right] \xi-(z+D-2)^{2}\right\} P_{5}(z ; \xi) \\
& \times \kappa \Phi^{4} /\left(64\left\{2(D-3)(D-4)(z+D-2) P_{4}(z) \beta_{3}-\left[3 z^{2}+(D+2)(D-2)\right] P_{3}(z) l^{2}\right\}\right)
\end{aligned}
$$

while the coupling constants and the cosmological one are tied as follows

$$
\begin{aligned}
\beta_{1} & =\left\{4\left[4 \tilde{P}_{6}(z) \xi-(z+D-2)^{2} \tilde{P}_{4}(z)\right] \beta_{3}+(z+D-2)\left[4 P_{3}(z) \xi-\tilde{\tilde{P}}_{3}(z)\right] l^{2}\right\} /\left[2(z+D-2) P_{5}(z ; \xi)\right], \\
\beta_{2} & =4\left\{(z-1)(z+D-2)\left(3 z^{2}+D^{2}-4\right) l^{2}-P_{6}(z ; \xi) \beta_{3}\right\} /\left[(z+D-2) P_{5}(z ; \xi)\right], \\
\lambda & =-\frac{1}{4 l^{2}}\left(2 z^{2}+(D-2)(2 z+D-1)-\frac{4(D-3)(D-4) z(z+D-2) \beta_{3}}{l^{2}}\right),
\end{aligned}
$$

where for simplicity we have defined

$$
\begin{aligned}
& P_{3}(z) \equiv 9 z^{3}-3(9 D-14) z^{2}-(D-2)(5 D-62) z-(D-2)\left(D^{2}-4 D+36\right), \\
& \tilde{P}_{3}(z) \equiv 6 z^{3}-4(5 D-8) z^{2}-3(D-2)(D-15) z-(D-2)\left(D^{2}-3 D+26\right), \\
& P_{4}(z) \equiv 27 z^{4}-36(3 D-5) z^{3}-2(D-2)(5 D-116) z^{2}-4(D-2)\left(D^{2}-D+30\right) z-(D+2)(D-2)^{3}, \\
& P_{5}(z ; \xi) \equiv 4\left[2 z^{2}+(D-2)(2 z+D-1)\right] P_{3}(z) \xi-(z+D-2)^{2} \tilde{P}_{3}(z), \\
& \tilde{\tilde{P}}_{3}(z) \equiv 15 z^{3}-(19 D-22) z^{2}-3(D-2)(D-18) z-(D-2)\left(D^{2}-4 D+36\right), \\
& \tilde{P}_{4}(z) \equiv 3 z^{4}-(7 D-10) z^{3}-\left(3 D^{2}-2 D-25\right) z^{2}-\left(D^{3}-14 D^{2}+30 D+4\right) z-(2 D+7)(D-2)^{2}, \\
& \tilde{\tilde{P}}_{4}(z) \equiv 9 z^{4}-6(3 D-4) z^{3}-8\left(D^{2}-10\right) z^{2}+2\left(D^{3}-4 D^{2}+32 D-80\right) z-(D-2)\left(D^{3}+2 D^{2}-12 D+24\right), \\
& \tilde{\tilde{P}}_{4}(z) \equiv 6 z^{4}-2(7 D-10) z^{3}-\left(13 D^{2}-53 D+34\right) z^{2}+2\left(D^{3}-D^{2}+D-16\right) z-(D-2)\left(D^{3}+D^{2}-10 D+20\right), \\
& P_{6}(z ; \xi) \equiv 4\left[2 z^{2}+(D-2)(2 z+D-1)\right] \tilde{\tilde{P}}_{4}(z) \xi-(z+D-2)^{2} \tilde{\tilde{P}}_{4}(z), \\
& \tilde{P}_{6}(z) \equiv 9 z^{6}-3(3 D-2) z^{5}-\left(2 D^{2}+90 D-275\right) z^{4}-2\left(11 D^{3}-46 D^{2}-17 D+172\right) z^{3}-(D-2)\left(7 D^{3}-62 D^{2}\right. \\
&+212 D-256) z^{2}-(D-2)(D-4)\left(D^{3}-8 D^{2}+34 D-76\right) z-\left(2 D^{2}+7 D-6\right)(D-2)^{3} .
\end{aligned}
$$

\section{APPENDIX C: PARAMETERS ASSOCIATED TO THE SECOND CLASS OF CRITICAL SOLUTIONS (24)}

The self-interaction which ensures the existence of the critical solution (24) is given by

$$
\begin{aligned}
U(\Phi)= & \frac{\tilde{P}_{2}(z ; \xi)}{4 l^{2}}\left(2 \Phi^{2}+[(2 z-D-2)(D-1) \xi-(z-1)(2 z-D-1)] l^{2} \kappa \Phi^{4} /\left[4(D-3)(D-4) z(z-D) \beta_{3}\right.\right. \\
& \left.\left.-(D-1)(2 z-D-2) l^{2}\right]\right),
\end{aligned}
$$

while the rest of the parameters are fixed as

$$
\begin{aligned}
\beta_{1}= & \left(\left\{4\left[3(D-2) z^{4}+\left(D^{2}-10\right) z^{3}+(D+2)(D-3)^{2} z^{2}-(D-2)\left(2 D^{2}-8 D+9\right) z-(D-2)(D-4)\right] \xi\right.\right. \\
& \left.\left.-4(z-1)^{2}\left[\left(D^{2}-D-3\right) z+D-4\right]\right\} \beta_{3}+(z-1)[(D-2)(3 z+D-4) \xi-(z-2)(D-1)] l^{2}\right) \\
& /\left[2(D-2)(z-1)(3 z+D-4) \tilde{P}_{2}(z ; \xi)\right], \\
\beta_{2}= & -\left(4 \left\{\left[2 z^{2}+(D-2)(2 z+D-1)\right]\left[6(D-2) z^{2}-\left(D^{2}-3 D+8\right) z-2 D+8\right] \xi\right.\right. \\
& \left.\left.-(z-1)^{2}[D(5 D-11) z+2(D-1)(D-4)]\right\} \beta_{3}-(D-1)(z-1)(2 z-D-2) l^{2}\right) \\
& /\left[2(D-2)(z-1)(3 z+D-4) \tilde{P}_{2}(z ; \xi)\right], \\
\lambda= & -\frac{1}{4 l^{2}}\left(2 z^{2}+(D-2)(2 z+D-1)-\frac{4(D-3)(D-4) z(z+D-2) \beta_{3}}{l^{2}}\right) .
\end{aligned}
$$




\section{APPENDIX D: EXTENSIVE COEFFICIENTS}

The extensive thermodynamic quantities of each presented solution are proportional to a mutual coefficient encompassing the details to which the theory is thermodynamically sensitive; i.e., the values of this coefficient determine all the points in the parameter space of the theory probed by the solution, which correspond to the same thermodynamic behavior. In this Appendix we present these extensive coefficients for all solutions. Since they usually take long expressions, this not only allows us to write concise expressions for the extensive thermodynamic quantities but also make evident the aforementioned proportionality, that incidentally is the base of the Smarr formula (20).

The dimensionless mutual coefficient appearing in the extensive thermodynamic quantities of the first class of the solutions of subsection IVA is given by

$$
\begin{aligned}
l^{2} \Upsilon_{1}= & \frac{(z+D-2)(3 z-D-2)}{4 P_{5}(z ; \xi)}\left(2 ( D - 3 ) ( D - 4 ) \left\{16(3 z+D-2)\left[2 z^{2}+(D-2)(2 z+D-1)\right] \xi\right.\right. \\
& \left.\left.-(5 z+3 D-6)(z+D-2)^{2}\right\} \beta_{3}-(z+D-2)\left[3 z^{2}+(D-2)(D+2)\right] l^{2}\right),
\end{aligned}
$$

where the polynomial $P_{5}(z ; \xi)$ was previously defined in Appendix B.

The dimensionless extensive coefficient related to the second class of the solutions of subsection IV B is written as

$$
l^{2} \Upsilon_{2}=\frac{8(D-2)(D-1)^{2}}{D+2} \beta_{1}-\frac{4\left(D^{3}-D^{2}-2 D-4\right)}{D+2} \beta_{3}-\frac{(D-1)(D-2)(1-4 \xi) l^{2}}{(D+2)[D-1-(5 D-2) \xi]} .
$$

Finally, the dimensionless extensive coefficient of the last class in Sec. VI is

$$
l^{2} \Upsilon_{3}=-\frac{2(D+1)(2 D-1) \sqrt{\alpha^{2}+4}}{P_{2}(D)\left(\alpha+\sqrt{\alpha^{2}+4}\right)},
$$

where the polynomial $P_{2}(D)$ is defined in the same section.

[1] S. Kachru, X. Liu, and M. Mulligan, Phys. Rev. D 78, 106005 (2008).

[2] E. A. Bergshoeff, O. Hohm, and P. K. Townsend, Phys. Rev. Lett. 102, 201301 (2009).

[3] E. Ayon-Beato, A. Garbarz, G. Giribet, and M. Hassaine, Phys. Rev. D 80, 104029 (2009).

[4] E. Ayon-Beato, A. Garbarz, G. Giribet, and M. Hassaine, J. High Energy Phys. 04 (2010) 030.

[5] T. Azeyanagi, W. Li, and T. Takayanagi, J. High Energy Phys. 06 (2009) 084.

[6] A. Giacomini, G. Giribet, M. Leston, J. Oliva, and S. Ray, Phys. Rev. D 85, 124001 (2012).

[7] M. Bravo-Gaete and M. Hassaine, Phys. Rev. D 89, 104028 (2014).

[8] F. Correa, M. Hassaine, and J. Oliva, Phys. Rev. D 89, 124005 (2014).

[9] F. Herrera and Y. Vsquez, Phys. Lett. B 782, 305 (2018).

[10] D. W. Pang, J. High Energy Phys. 01 (2010) 116.

[11] A. Alvarez, E. Ayon-Beato, H. A. Gonzalez, and M. Hassaine, J. High Energy Phys. 06 (2014) 041.

[12] M. Taylor, arXiv:0812.0530.

[13] J. Tarrio and S. Vandoren, J. High Energy Phys. 09 (2011) 017.
[14] M. Bravo-Gaete and M. Hassaine, Phys. Rev. D 91, 064038 (2015).

[15] M. K. Zangeneh, A. Sheykhi, and M. H. Dehghani, Phys. Rev. D 92, 024050 (2015).

[16] H. A. Gonzalez, D. Tempo, and R. Troncoso, J. High Energy Phys. 11 (2011) 066.

[17] E. Ayón-Beato, M. Hassaïne, and M. M. Juárez-Aubry, Phys. Rev. D 90, 044026 (2014).

[18] E. Ayon-Beato, M. Bravo-Gaete, F. Correa, M. Hassaine, M. M. Juarez-Aubry, and J. Oliva, Phys. Rev. D 91, 064006 (2015).

[19] E. Shaghoulian, Phys. Rev. D 93, 126005 (2016).

[20] E. Shaghoulian, Phys. Rev. D 94, 104044 (2016).

[21] M. B. Gaete, L. Guajardo, and M. Hassaine, J. High Energy Phys. 04 (2017) 092.

[22] V. Iyer and R. M. Wald, Phys. Rev. D 50, 846 (1994).

[23] W. Kim, S. Kulkarni, and S.-H. Yi, Phys. Rev. Lett. 111, 081101 (2013).

[24] Y. Gim, W. Kim, and S. H. Yi, J. High Energy Phys. 07 (2014) 002.

[25] L. F. Abbott and S. Deser, Nucl. Phys. B195, 76 (1982); Phys. Lett. 116B, 259 (1982); S. Deser and B. Tekin, Phys. Rev. Lett. 89, 101101 (2002); Phys. Rev. D 67, 084009 (2003). 
[26] L. Smarr, Phys. Rev. Lett. 30, 71 (1973); 30, 521 (1973).

[27] H. Lu and C. N. Pope, Phys. Rev. Lett. 106, 181302 (2011).

[28] S. Deser, H. Liu, H. Lu, C. N. Pope, T. C. Sisman, and B. Tekin, Phys. Rev. D 83, 061502 (2011).
[29] G. Anastasiou, R. Olea, and D. Rivera-Betancour, Phys. Lett. B 788, 302 (2019).

[30] W. G. Brenna, R. B. Mann, and M. Park, Phys. Rev. D 92 , 044015 (2015).

[31] G. W. Horndeski, Int. J. Theor. Phys. 10, 363 (1974). 